\title{
TTR
}

Traduction, terminologie, re?daction

\section{"Le soleil a rendez-vous avec la lune... " ou des problèmes posés par le genre dans la traduction vers le français}

\section{Christine Klein-Lataud}

Volume 9, numéro 2, 2e semestre 1996

Parcours de traduction

Pathways of Translation

URI : https://id.erudit.org/iderudit/037262ar

DOI : https://doi.org/10.7202/037262ar

Aller au sommaire du numéro

Éditeur(s)

Association canadienne de traductologie

ISSN

0835-8443 (imprimé)

1708-2188 (numérique)

Découvrir la revue

Citer cet article

Klein-Lataud, C. (1996). «Le soleil a rendez-vous avec la lune... » ou des problèmes posés par le genre dans la traduction vers le français. TTR, 9(2), 147-164. https://doi.org/10.7202/037262ar
Résumé de l'article

" Le soleil a rendez-vous avec la lune " ou des problèmes posés par le genre dans la traduction vers le français - Après un bref exposé des données linguistiques concernant le genre grammatical, cet article se propose d'explorer les problèmes liés à l'expression du genre et du sexe dans la traduction des textes littéraires. Il illustre d'abord l'influence du genre sur le travail de l'imaginaire, puis, à partir d'une nouvelle de Ruth Rendell, il montre combien l'obligation d'exprimer le sexe en français alors que l'anglais peut rester ambigu (the lover = l'amant ou l'amante) rend difficile l'entretien du suspense policier. Enfin, la traduction d'un poème d'Erin Mouré fournit l'occasion d'examiner d'autres exemples des difficultés qui surviennent quand on traduit de l'anglais vers une langue comme le français dont tous les substantifs sont affectés d'un genre.
Tous droits réservés @ C TTR: traduction, terminologie, rédaction — Les auteurs, 1996
Ce document est protégé par la loi sur le droit d'auteur. L’utilisation des services d'Érudit (y compris la reproduction) est assujettie à sa politique d'utilisation que vous pouvez consulter en ligne. 


\section{"Le soleil a rendez-vous avec la lune... " ou des problèmes posés par le genre dans la traduction vers le français}

\section{Christine Klein-Lataud}

La tradition de l'analyse grammaticale scolaire nous a habitués à mettre sur le même plan la notion de nombre et celle de genre. Fausse symétrie, puisque ces deux traits grammaticaux ne sont pas du tout de même nature. J'en emprunterai la démonstration à André Martinet.

Le genre grammatical est une des catégories linguistiques qui structurent certaines langues telles que les langues romanes et slaves. C'est simplement un élément formel dénué de pertinence sémantique.

Le genre féminin appartient [...], d'emblé et exclusivement, à la morphologie. Il désigne une série de modifications formelles entraîntes par le choix de tel nom au lieu de tel autre, chaise, par exemple, au lieu de fonteull. [...] On ne peut pas poser un monème de genre féminin, parce que les différences formelles qui manifestent le genre feminin ne correspondent à aucun choix de sens distinct de celui du nom (Martinet, 1970, p. 12).

Autrement dit, à la différence du nombre, qui est choisi en fonction du message (Voici un exemple / Voici des exemples), le 
genre du mot est imposé au locuteur par la langue (un exemple / *une exemple).

Mais « ce qui complique l'affaire est que la différence entre les formes "masculine" et "féminine" de l'adjectif est, fréquemment, dans les langues à genre féminin, utilisé comme élément de dérivation pour distinguer les êtres de sexe féminin des êtres de sexe masculin : on distingue entre l'épicier et l'épicière de la même façon qu'entre la forme "masculine" de l'adjectif premier et sa forme "féminine" première. " (Martinet, 1985, pp. 55-56) Les locuteurs ont donc tendance à confondre le " genre arbitraire " des êtres qui ne se distinguent pas en opposition (la langue impose la chaise, le fauteuil : il n'y a pas de *le chaise, *la fauteuil fonctionnant en opposition avec la chaise, le fauteuil qui offrirait un choix aux locuteurs) avec l'opposition sexuée, et à le doter d'un sens. C'est ce type d'interprétation que certains linguistes appellent " sexuisemblance ". Par exemple, ce ne serait pas un hasard si le mot chaise est affecté du genre féminin alors que le mot fouteuil est du genre masculin : la répartition des genres exprimerait un rapport de force. À la modeste chaise, le féminin ; au majestueux fauteuil, le masculin. Certains grammairiens d'autrefois ont ainsi expliqué le changement de genre du mot orgue : " n'est-ce pas l'idée de puissance, de génie qui prive souvent ce nom de la féminité que sa terminaison lui destine ? " I) fallait le masculin pour traduire " l'idée de chef d'ceuvre' ! " Il reste que pour les linguistes, il est abusif d'accorder une signification au genre d'un nom.

Aux yeux des poètes, toutefois, il en va tout autrement, et quand le soleil a rendez-vous avec la lune, le masculin de soleil et le féminin de lune esquissent déjà tout un programme narratif. L'idylle est là, prête à naître. Car le genre grammatical est inclus dans la vaste entreprise de remotivation du signe qui préside à l'entreprise littéraire. De même qu'en jouant sur le phonétisme des

1. Louis-Nicolas Bescherelle, Henri Bescherelle et Litais De Gaux, Grammaire nationale (Paris, Garnier Frères, 1864; $12^{\circ}$ éd.), cités par Céline Labrosse (1996, p. 25). 
mots à l'intérieur d'un texte, les écrivains vont à rebours de l'arbitraire du signe linguistique et instaurent un lien de motivation entre signifiant et signifié, de même ils peuvent utiliser le genre, pure catégorie morphologique, pour l'affecter d'une valeur sémantique.

C'est vers Gaston Bachelard, ce " rêveur de mots ", que je me tournerai pour éclairer les effets indus et délicieux du genre des mots. Dans sa Poétique de la rêverie, il s'interroge en phénoménologue sur la naissance de la rêverie poétique et voit dans le genre des mots une des sources de l'imaginaire : " comment [le philosophe songeur] ne serait-il pas sensible à la rivalité du masculin et du féminin qu'il découvre à l'origine de la parole ? " (Bachelard, 1984 , p. 25) Ainsi, l'opposition entre le rêve et la rêverie lui semble refléter la division de la psyché entre animus et anima, et il voit dans le genre féminin un moyen d'investir le noyau de la rêverie féminine. (Rappelons, pour éviter tout malentendu, que masculin et féminin renvoient non au sexe biologique mais à une partie de l'âme humaine présente dans chaque individu.)

En rêvant à notre langue maternelle, dans notre langue materneile [...] nous croyons déjà reconnâttre un privilège de rêverie aux mots féminins. Déjà les désinences féminines ont de la douceur. [...] Des heures et des heures, dès que j'ai eu le bonheur d'avoir un dictionnaire, je me laissais séduire par le féminin des mots. Ma réverie suivait les inflexions de la douceur. Le féminin dans un mot accentue le bonheur de parler. (Bachelard, 1984, pp. 26-27)

L'interprétation du genre féminin comme porteur des valeurs traditionnelles de la féminité devient ainsi une façon de redonner aux mots leur puissance onirique.

On peut, ici, s'interroger sur l'expérience des enfants bilingues, dans l'esprit desquels se développent concurremment deux " visions du monde ». Élevé en français par sa grand-mère Charlotte, Andreï Makine, dans sa première enfance, passe sans conflit d'une langue a l'autre, malgré les changements de genre des mots renvoyant au même référent. « Enfant, je me confondais avec 
la matière sonore de la langue de Charlotte. J'y nageais sans me demander pourquoi ce reflet dans l'herbe, cet éclat coloré, parfumé, vivant, existait tantôt au masculin et avait une identité crissante, fragile, cristalline imposee, semblait-il, par son nom de tsevok, tantôt s'enveloppait d'une aura veloutée, feutrée et féminine devenant une fleur. " (Makine, 1995, pp. 243-244) Le genre participe de l'être des mots auxquels les poètes, à la suite de Claude Esteban, prêtent la faculté « d'épouser la substance des choses et de la rendre comme translucide dans la profération verbale qu'ils nous permettent. " (Esteban, 1990, p. 30) La rêverie suscitée par le mot est donc différente si le genre du mot change ou disparaít lors de la traduction.

Le rêveur de Bachelard, bien installé dans sa vision sexuée de l'univers et dans sa "génosanalyse " (c'est-à-dire analyse en termes de genre) des textes littéraires, ne peut qu'être ébranlé lors de cette radicale métamorphose. "Quel trouble alors quand, passant d'une langue à une autre, on a l'expérience d'une féminité perdue ou d'une féminité masquée par des sons masculins ! " C'est ainsi que Bachelard s'avoue incapable de rêver aux textes cosmiques allemands en raison de " l'extraordinaire inversion qui donne au soleil le genre féminin et à la lune le genre masculin. Quand la discipline grammaticale oblige des adjectifs à se masculiniser pour s'associer à la lune, un rêveur français a l'impression que sa rêverie lunaire se pervertit ${ }^{2}$. " (Bachelard, 1984, p. 28) Et encore, dans ce cas, la double inversion, si gênante qu'elle puisse être pour un locuteur français, conserve l'opposition des genres. Mais si le passage d'une langue à l'autre l'abolit, la perte de sens est grave. En témoigne le poème d'Henri Heine évoquant le sapin solitaire, sommeillant sous la neige et la glace, et rêvant d'un palmier qui se désole dans l'Orient lointain et brâlant : en allemand, l'opposition Nord / Sud symbolisée par les deux arbres se double d'un symbolisme amoureux puisque sapin est masculin, et que paimier est

2. Dans le même ordre d'idtes, on peut se demander comment traduire en allemand I'Imitation de Notre-Dame la Lune de Jules Laforgue. 
féminin. Mais en français, comment le palmier pourrait-il évoquer une « sirène des sables »? Un phénomène analogue d'abolition de l'opposition des genres se produit en russe, et Roman Jakobson, lisant la traduction de ce poème par Lermontov, se dit mal à l'aise parce que les deux arbres sont du genre féminin en russe. Voilà un des cas où le traducteur est irrémédiablement prisonnier de la langue.

Quelquefois, le symbolisme est plus diffus, mais imprègne néanmoins le texte littéraire. Dans sa communication "Une éducation sentimentale : Approfondissement de la théorie interliminale de la traduction et du goût post-moderne», Marilyn Gaddis Rose comparait une description de Flaubert avec sa traduction en anglais et soulignait le rôle de la " sexualité secrète des genres grammaticaux françajs ». «La nature de l'anglais impose la suppression de tout anthropomorphisme, par exemple, pas de lopin herbeux masculin (un herbage) allant rejoindre une petite butte féminine (une colline), pas de moulin pré-freudien entouré par de la verdure broussailleuse. " (Gaddis Rose, à paraître)

Une langue sans genre, comme l'anglais, peut, par le jeu de la personnification, opérer une catégorisation sexuelle d'un inanimé. Et une certaine liberté existe dans le choix du sexe, malgré les traditions culturelles. Ainsi, le soleil est généralement masculin en anglais quand il est personnifié, mais je l'ai trouvé au féminin dans un texte de Karen Blixen. " As I looked round to the opposite horizon, the sun just came up, dull and red. [...] In three ou four hours she would be white-hot, and fierce upon the head of the wanderer. " (Blixen, 1984, p. 145) Le choix de l'auteure vient sans doute du désir de prêter une identité féminine à la funeste divinité, la Furie qui va s'acharner sur l'infortuné voyageur parti à pied dans la savane africaine...

Parmi les grandes entités hantant la psyché humaine, la mort est une des plus importantes. En français, elle est symétrique de la vie, ces deux déesses présidant a notre destinée. Vu dans la perspective des rêveries bachelardiennes, le féminin favorise, me 
semble-t-il, l'euphémisation, rend la mort plus facilement maternelle, consolatrice, voire amoureuse. Pensons à Gérard de Nerval :

"Celle que j'aimai seul m'aime encore tendrement

C'est la Mort - ou la Morte... ô délice ! 6 tourment ! » (G. de Nerval, 1960, p. 5)

La mort rejoint la Nature, la Terre, la Nuit parmi les forces féminines ambivalentes mais souvent favorables. En anglais, la mort, conventionnellement représentée au masculin (ex. : " Death is a beautiful naked man " (L. de Bernières, 1996, p. 140)), peut a l'occasion se féminiser (se travestir ?) comme dans les nouvelles d'Edgard Poe. Mais dans une langue à genre, comme l'allemand, qui lui affecte le genre masculin, la Mort ne peut plus apparaître comme une présence féminine consolatrice et les images sont irrémédiablement altérées.

Pour respecter la connotation symbolique du genre, le traducteur peut parfois ruser. J'emprunterai mon premier exemple à Claire Cayron, traductrice attitrée du grand écrivain portugais Miguel Torga. Dans Sésame, pour la traduction, elle souligne que l'écriture littéraire exige une attention sans relâche aux micro-structures, dont fait partie le genre grammatical. Ainsi, toute la signification de la nouvelle " Le Secret » repose, selon elle, sur le choix d'un mot féminin pour désigner le lieu de bajgnade du jeune Pedro. Le portugais a lagoa, féminin, signifie « le lac ", "l'étang ". Or " tout doit être féminin dans l'atmosphère du jeune "homme", et même le liquide où il retourne avant de mourir. Le sens symbolique de la baignade est irrémédiablement perdu si au lieu de « la lagune elle-même, lorsqu'il s'y mira, le troubla dans un premier temps. Mais c'était la lagune, sa vieille amie... ", on traduit "l'étang lui-même, lorsqu'il s'y mira, le troubla dans un premier temps. Mais c'était l'étang, son vieil ami".[...] Dans cette formulation, il n'y a plus de "secret" formel, plus de littérature. " (Cayron, 1987, p. 169) La solution de Claire Cayron, c'est-à-dire le choix de " lagune ", seul mot féminin disponible en français, s'écarte du signifié du portugais, mais respecte les connotations 
engendrées par le signifiant, qui sont ce qui importe le plus en ce cas.

Autre exemple, emprunté cette fois au japonais. Dans une de ses nouvelles, Dazai Osamu raconte l'histoire d'un billet de cent yens. Le japonais est une langue sans genre grammatical, mais en exergue, Dazai Osamu en assigne un au billet, et le présente dans son récit comme une entité féminine passant de main en main, de plus en plus usée et épuisée. L'exergue affirme avec désinvolture : "Dans les langues étrangères, tous les noms ont un genre. Ainsi, je ferai de l'argent un féminin ". Donc, aussi bien la déclaration explicite de l'auteur que la dynamique textuelle de personnification du billet exigent un féminin, alors que billet en français est un masculin. Dans ce cas, la traductrice française a eu recours au mot " coupure " ("Souvenirs d'une coupure de cent yen ", D. Osamu, 1986). Pour opérer la féminisation du billet-narratrice, le traducteur anglais, lui, a da utiliser des moyens indirects comme la précision " female " (" I now worked as a female runner on the black market », D. Osamu, 1983, p. 207), de même qu'il a adapté l'exergue ( Certain foreign languages distinguish between masculine and feminine nouns. Words for currency are feminine $»)$.

L'absence de genre neutre en français pose parfois des problèmes quand on traduit de l'anglais. En effet, l'opposition animé /inanimé peut être exploitée à des fins littéraires. Comme pour de nombreux jeux de langage, nous en trouvons un bel exemple dans Alice au pays des merveilles. Il se situe au chapitre sept, celui du the fou réunissant Alice, le Chapelier, le Loir et le Lièvre de mars. Alors qu'Alice utilise le mot " time " avec sa valeur de nom commun (minuscule à l'initiale, genre neutre), ses interlocuteurs le personnifient en lui mettant une majuscule et en le transformant en masculin : " I think you might do something better with the time [...] than wasting it in asking riddles that have no answers. " « If you knew Time as well as I do, said the Hatter, you wouldn't talk of wasting it. It's him . " Alice poursuit en disant : " I know that I have to beat time when I learn music. " (L. Carroll, 1970, p. 96) À quoi le Chapelier répond que cela explique tout puisque : " He won't stand beating." Les traducteurs français, privés de 
l'opposition animé / inanimé véhiculée par le neutre, doivent inventer une solution qui la remplace sémantiquement. Jacques Papy explicite : « Si tu connaissais le Temps aussi bien que moi, dit le Chapelier, tu ne parlerais pas de le perdre. Le Temps est un être vivant. [...] Tout ce que je sais, c'est qu'il faut que je batte les temps quand je prends ma leçon de musique [...] Ah ! ca explique tout. Le Temps ne supporte pas d'être battu. " (L. Carroll, 1977, p. 111) Le même problème se pose pour la traduction d'un passage de The Handmaid's Tale de Margaret Atwood. Au moment de tenter de fuir le pays en proie à la dictature, Luke et la narratrice passent en revue leur plan pour s'assurer qu'ils n'ont oublié aucun détail. Soudain, Luke s'exclame " The cat ". Sa femme lui répond "We could just leave her " mais se rend compte que c'est impossible parce que les miaulements attireraient l'attention des voisins. Luke clôt la discussion en disant " I'll take care of it " (M. Atwood, 1985 , p. 202) et la narratrice commente : « because he said it instead of her, I knew he meant kill. That is what you have to do before you kill [...] You have to create an it ". Voici la solution retenue par la trađuctrice, Sylviane Rué : « Je vais m'occuper de ça. Et parce qu'il avait dit ça au lieu de lui, je sus qu'il voulait dire tuer.[...] Il faut créer un ça, là où il n'y en avait pas auparavant. » (M. Atwood, 1987, p. 231) En fait, le commentaire métalinguistique porte à faux parce que le ça de « Je vais m'occuper de ça » renvoie à l'acte de tuer lui-même et non au chat. Personnellement, j'aurais plutôt explicité lexicalement la valeur du neutre anglais, exprimant ici l'opposition humain / non-humain ( Je vais m'occuper de cette bête. Et parce qu'il avait dit cette bête au lieu d'elle, je sus qu'il voulait dire tuer.)

L'opposition des genres masculin / féminin recoupe à l'occasion un contraste, sémantique celui-là, entre les sexes. Exemple, le suffixe français -esse (prince / princesse). Or l'anglais a de nombreux nóms référant à des animés qui ne portent pas d'indication de sexe, alors que le français est très souvent obligé d'indiquer s'il s'agit d'un homme ou d'une femme. Le choix traductionnel peut s'avérer délicat, comme lorsque Harold Nicolson écrit à sa femme qu'il vient de rencontrer a charming hairdresser : un coiffeur ou une coiffeuse? Sa biographie nous indique que le 
premier choix est le bon. L'anglais permet une grande discrétion ( $m y$ friend, my companion, my lover), et seule une éventuelle reprise par un pronom révelera le sexe de la personne, alors que le français est obligé de préciser ami / amie, compagnon / compagne, amant / amante. Jakobson (1963) observait à juste titre que les langues different moins par ce qu'elles permettent d'exprimer que par ce qu'elles sont obligées d'exprimer. Le passage d'une langue à l'autre peut donc s'avérer problématique. Ainsi, dans le roman de David Ladge Paradise News (Date?, pp. 70 et 252), un couple de petits bourgeois anglais part à Hawaï à l'invitation de leur fils. Ce dernier, qui vit en Australie, doit les rejoindre en compagnie de $a$ very special friend qu'il brûle de leur présenter. Bonheur des parents qui échafaudent déjà des projets matrimoniaux et attendent avec impatience la rencontre : "I expect she'll be at the airport with him. " Surprise, surprise. La mère, effondrée, écrit à sa fille : " His "special friend" turned out to be a man. " L'indétermination est difficile à maintenir en français.

Une nouvelle de Ruth Rendell, "The Wrong Category ", repose entièrement sur cette mancuure d'escamotage du sexe permise par la morphologie anglaise. Une série de meurtres dévaste une communauté anglaise. Les victimes appartiennent toutes à la même « catégorie » et répondent à la même description (large liquid eyes, full soft mouth, long dark hair, doe-eyed, girlish faces). Le héros, fasciné par les meurtres, passe en revue les victimes précédentes et s'amuse à essayer de deviner la victime suivante en hantant les bars et les lieux dangereux. $\AA$ la fin de l'histoire, on le voit tard dans la nuit s'engager avec une jeune femme dans une ruelle sinistre. On pressent qu'il va y avoir un meurtre, et c'est effectivement ce qui se produit. Seulement, à l'inverse de ce qu'on attend, c'est la femme qui est la meurtrière... Et toutes les victimes étaient des hommes, du genre efféminé. Ambiguitté du mot " category ": le héros n'était pas du bon " genre " (la meurtriére a toujours choisi des jeunes hommes efféminés), mais contrairement à ce que croyaient les lecteurs, il était du bon sexe ! Le paradigme des désignations anglaises permet de ne jamais indiquer le sexe (the victim, the one they called Pat Leston, the Italian one, a candidate, one of them; the maniac, the murderer, the killer) et tout concourt 
à faire croire que les victimes sont des jeunes femmes : les éléments descriptifs, clichés de la féminité version Harlequin ; l'adjectif girlish, interprété comme " đe jeune fille, enfantine " à la première lecture, qui sera réinterprété après coup comme " efféminé "; le diminutif Pat, perçu comme celui de Patricia, qui s'avérera celui de Patrick. La traduction française doit, bien entendu, faire naître la même méprise, mais c'est plus difficile. Obligé, obligée ( ! ) par la langue de choisir entre le candidat et la candidate, l'Italien et l'Italienne, celui ou celle, comment faire ? S'accrocher aux mots qui ne sont pas sexués, comme la victime et l'assassin, et y ancrer les anaphoriques (la victime italienne, celle-ci, etc.). On remarquera, au passage, que le genre des mots " assassin " et " victime " n'est peut-être pas lui-même un pur accident linguistique !

Ma dernière illustration des difficultés posées par le passage d'une langue comme l'anglais à une langue comme le français, où tous les substantifs sont affectés d'un genre, sera la traduction d'un poème d'Erin Mouré (1995). Tricks ${ }^{3}$ est écrit dans une langue apparemment simple, et une fois réglés les habituels problèmes de registre, de rythme et de musicalité, la traduction me semblait devoir être elle aussi très simple. Or il n'en a rien été. En fait, elle m'a donné un mal de chien (de chienne ?) Mes difficultés ont été provoquées par les problèmes de sexe et de genre. Le premier mot à faire problème est $d o g$. En soi, $d o g$ ne révèle pas s'il s'agit d'un chien ou d'une chienne. Il existe bien un féminin en anglais, bitch, mais il est d'un usage soit technique (vocabulaire des éleveurs), soit argotique (insulte pour une femme, équivalent de salope ou de garce). La dédicace : " for Trix, $a$ dog » laisse donc dans l'ombre le sexe du chien. Au début du poème, a dog est repris par le pronom neutre : "... it remembers its young doghood ». Mais plus loin, my old dog est repris par le pronom féminin «She sleeps on the porch... she rests "). On est donc contraint, en français, d'unifier et de recourir au féminin dans l'ensemble du poème. Se pose alors le probleme de la traduction de doghood (her old / young doghood). Car la vie d'un chien n'est pas forcément une vie de chien. On est

3. Voir le texte complet et sa traduction en appendice. 
sauvé ici par une vie de chienne, qui évite la confusion avec l'idiotisme... Plus loin, le poème mentionne $a$ black pup avec lequel la chienne se promène au temps de sa jeunesse. Or, pup est également repris par le pronom féminin ( who was herself splay-legged on the fireroad "). La définition de "chiot ", n. m., est " jeune chien ", ce qui est donc contraire aux indications du texte : il s'agit d'une petite chienne amie, et non d'un chien copain ou soupirant. Mais comment forger le féminin correspondant, qui serait " chiotte " ? Les deux mots ont une étymologie différente (chiot vient du latin dialectal catellus, chiottes vient du latin cacare), mais hélas, par un malheureux accident de la langue, ils se rencontrent dans la manifestation de surface. Force est donc de passer par l'inexactitude du mot " chiot » ou d'avoir recours à la périphrase " jeune chienne ». Les choses se compliquent encore avec le mot god. Exprimant son sentiment de déréliction, l'énonciatrice dit : "I feel I am in the world \& there is no god in it with me. " La divinité est traditionnellement en Occident du sexe mâle, même si certaines églises protestantes s'efforcent de remplacer l'image d'une divinité masculine par une sorte d'entité bisexuée (Dieu le père-la mère). C'est Dieu qu'on invoque, c'est Dieu dont on déplore la mort. On parle d'un monde sans Dieu, pas d'un monde sans Déesse. Or, dans le poème, c'est la chienne qui est assimilée à Dieu. Dans la montagne, elle règne « like a god ». Si l'on traduit par « telle une déesse ", en conformité avec le sexe de la chienne, on oblitère le lien entre le dieu dont on regrette l'absence au début et la chienne, identification que confirme la strophe finale: "Without her memories I am alone in the world, the god gone out of it. ". Mieux vaut donc traduire as a god par " telle un dieu ", la bizarrerie grammaticale étant moins grave que la perte de sens majeure qui se produirait si on ne dégageait pas le lien god / dog (qui, par parenthèse, sont des palindromes : on touche ici aux limites de la traduisibilite !).

Dans le même poème, le mari alcoolique assimile les femmes à des verres de whisky. " He says there are glasses as big as women filled with rye \& he wants to marry one. " L'absence de genre permet à l'anglais de rester équivoque : le one que veut épouser le mari représente-t-il le verre ou la femme ? Sans doute les 
deux à la fois, confondus dans son rêve d'alcoolique. Une pirouette permet de conserver l'équivoque en français : il suffit de remplacer le masculin " un verre " par le féminin « une chope ", malgré l'impropriété du terme.

Il apparaît en définitive que si certains problèmes de traduction restent insolubles (en particulier la détermination symbolique du sexe par le genre lors du processus de personnification d'un inanimé), d'autres peuvent être contournés ou résolus par l'ingéniosité des traducteurs, auxquels il appartient alors... de savoir décrocher la lune.

\section{Université York}

\section{Bibliographie}

ATWOOD, Margaret (1985). The Handmaid's Tale. Toronto, McClelland and Stewart.

(1987). La Servante Écarlate, trad. de Sylviane Rué. Paris, Robert Laffont, coll. « Pavillons ".

BACHELARD, Gaston (1984). La Poétique de la rêverie. Paris, PUF ; $1^{\text {bै }}$ éd. : 1960.

BERNIĖRES, Louis de (1996). Captain Corelli's Mandolin. Londres, Minerva.

BLIXEN, Karen (1984). Out of Africa. New York, Penguin.

CARROLL, Lewis (1970). Alice's Adventures in Wonderland. Londres, Penguin.

(1977). Alice au pays des merveilles, traduction de Jacques Papy. Paris, Régine Desforges. 
CAYRON, Claire (1987). Sésame, pour la traduction. Éd. Le Mascaret.

ESTEBAN, Claude (1990). Le Partage des mots. Paris, Gallimard, coll. " L'un et l'autre".

GADDIS ROSE, Marilyn (à paraître). " Une éducation sentimentale : Approfondissement de la théorie interliminale de la traduction et du gout post-moderne ", colloque La Traduction au sein des sciences humaines de l'Association canadienne-française pour l'avancement des sciences, Montréal, Univ. McGill, 17 mai 1996.

JAKOBSON, Roman (1963). « La Notion de signification grammaticale selon Boas ", Essais de linguistique générale, traduit de l'anglais et préfacé par Nicolas Ruwet. Paris, Minuit, coll. « Points ».

LABROSSE, Céline (1996). Pour une grammaire non sexiste. Montréal, Éd. du remue-ménage.

LODGE, David (1991). Paradise News. Londres, Secker and Warburg.

MAKINE, Andrer (1995). Le Testament français. Paris, Mercure de France.

MARTINET, André (1970). Grammaire fonctionnelle du français. Paris, Didier.

(1985). Syntaxe générale. Paris, Colin.

MOURÉ, Erin (1995). « Tricks », Ellipse, $\mathrm{n}^{\circ} 53$.

NERVAL, Gérard de (1960). "Artémis 》, les Chimères. Paris, Gallimard, coil. « La Pléiade ». 
OSAMU, Dazai (1983). Selected Stories and Sketches, translated by James O'Brien, China-Japan Program. Ithaca, Cornell University.

(1986). "Souvenirs d'une coupure de cent yen ", trad. par Jacqueline Pigeot. Les Ailes. La Grenade. Les Cheverax blancs et douze autres récits (1945-1960) (tome 2), nouvelles japonaises traduites du japonais par le groupe Kirin. Paris, Picquier.

\section{Appendice}

\section{Tricks}

for Trix, a dog

This is a life in which

a case of whisky is one drink.

In it, a dog goes totally blind \& no one knows

if it remembers its young doghood,

the smell of wild mountains carried in storm

from the high passes

I feel I am in the world \& there is no god in it with me.

These days my husband gets up \& sits

on the edge of our bed \& says

a case of whisky is one drink.

He says there are glasses as big as women filled with rye \&

he wants

to marry one.

This is what I listen to, no wonder

I can't sleep.

Faintly

I hear the heart-tick of my old dog in Calgary, 800 miles

away.

She sleeps on the porch, \& shies away when the footsteps 
come, crying gently.

When there are no footfalls, she rests \& waits to die.

I want to leave my husband \& let him marry

all the bottles in Vancouver,

while I go to Calgary to sit beside the blind dog of the

her eyes muddy with cataract,

family,

$\&$ tell her of her old/young doghood, of hikes to the

with a black pup in ' 71 , who was herself

ice-caves splay-legged on the fireroad.

I want to tell her she is a dog who loved the mountains, \& she should be proud even in blindness

that she saw them \& climbed their hard trails, \& camped there with the humans

like a god.

Now she is only afraid, of being stepped on.

She knows our voices, even mine that she hears so seldom. She speaks back in her small voice \& snuffles nearer.

I wish she would remember $\&$ be proud, but she lives only the present in her dogged blind way, fighting the back stairs.

Without her memories I am alone in the world, the god

My husband murmurs over, the root is still there, gone out of it. in the whole world there is only whisky for one drink. No wonder I can't sleep.

No wonder to look at the world is to go blind in it.

(Wanted alive)

Erin Mouré 


\section{Trucs}

Pour Trix, une chienne

C'est une vie où

une caisse de whisky ne fait qu'un seul verre.

Une vie où une chienne devient complètement aveugle

et où personne ne sait

si elle se souvient de son enfance de chiot,

de l'odeur des montagnes sauvages charriée par la tempête

à travers les hautes passes.

J'ai le sentiment d'être au monde sans qu'aucun dieu m'y tienne compagnie.

Ces jours-ci, mon mari s'assied sur le bord de notre lit et dit qu'une caisse de whisky, ça ne fait qu'un seul verre.

Il dit qu'il y a des chopes aussi grandes que des femmes pleines de

et qu'il veut en épouser une.

rye

Voilà ce que j'écoute, pas étonnant

si je ne peux pas dormir.

Faiblement

j'entends battre le coeur de ma vieille chienne à Calgary,

à huit cents milles. d'ici.

Elle dort sur la galerie, s'effarouche à l'approche des pas

et gémit doucement.

Quand nul pas ne résonne, elle se repose et attend la mort.

Je veux quitter mon mari et le laisser épouser

toutes les bouteilles de Vancouver.

Moi, j'irai à Calgary retrouver la chienne aveugle de notre famille, aux yeux envasés par la cataracte,

et je lui raconterai sa vie de chienne, vieille et jeune, ses excursions dans les grottes de glace

en 71 , avec un chiot noir qui a fini

écrasé sur la route.

Je veux lui raconter :

c'est une chienne qui aimait les montagnes, elle a beau être aveugle, elle devrait être fière 
de les avoir vues, d'avoir grimpé leurs rudes sentiers pour camper là-haut avec les humains telle un dieu.

Maintenant, elle n'est plus que peur, peur qu'on lui marche dessus. Elle reconnaît nos voix, même la mienne qu'elle entend si rarement. Elle répond de sa petite voix et s'approche en reniflant.

Je voudrais qu'elle se souvienne et qu'elle soit fière, mais elle ne vit que dans l'instant, aveugle et obstinée, avec pour tout combat les marches d'escalier.

Privée de ses souvenirs, je suis seule au monde, le dieu l'a déserté.

Mon mari continue à murmurer, les racines sont toujours là, tout le whisky du monde, ca ne fait qu'un seul verre.

Pas étonnant si je ne peux pas dormir.

Pas étonnant si regarder le monde, c'est devenir aveugle.

Traduit par Christine Klein-Latoud

RÉSUMÉ : «Le soleil a rendez-vous avec la lune » ou des problèmes posés par le genre dans la traduction vers le français - Après un bref exposé des données linguistiques concernant le genre grammatical, cet article se propose d'explorer les problèmes liés à l'expression du genre et du sexe dans la traduction des textes littéraires. Il illustre d'abord l'influence du genre sur le travail de l'imaginaire, puis, à partir d'une nouvelle de Ruth Rendell, il montre combien l'obligation d'exprimer le sexe en français alors que l'anglais peut rester ambigu (the lover = l'amant ou l'amante) rend difficile l'entretien du suspense policier. Enfin, la traduction d'un poeme d'Erin Mouré fournit l'occasion d'examiner d'autres exemples des difficultés qui surviennent quand on traduit de l'anglais vers une langue comme le français dont tous les substantifs sont affectés d'un genre. 


\begin{abstract}
Le soleil a rendez-vous avec la lune" or Problems Relating to Gender in Translating Towards French After a brief survey of the linguistic data concerning grammatical gender, this article explores the problems related to the expression of gender and sex in the translation of literary texts. First we look at the influence of gender on the imagination. Then, taking a short story by Ruth Rendell as an example, we show how the obligation to express the sex of protagonists in French can make it difficult to maintain suspense in a detective novel. Whereas English can keep a certain ambiguity ("the lover" can be either male or female), French is obliged to specify. Finally, the translation of a poem by Erin Mouré provides other examples of difficulties which arise translating from English into a language such as French, in which nouns have gender.
\end{abstract}

Christme Klein-Lataud : Collège universitaire Glendon, Université York, 2275, av. Bayview, Toronto (Ontario) M4N 3M6 CDN. Courriélec : Klataud@venus.yorku.ca 